Purdue University Purdue e-Pubs

2006

\title{
Direct Simulation of Transport in Open-Cell Metal Foams
}

S. Krishnan

J. Y. Murthy

S V. Garimella

Purdue University, sureshg@purdue.edu

Follow this and additional works at: http://docs.lib.purdue.edu/coolingpubs

Krishnan, S.; Murthy, J. Y.; and Garimella, S V., "Direct Simulation of Transport in Open-Cell Metal Foams" (2006). CTRC Research Publications. Paper 269.

http://dx.doi.org/10.1115/1.2227038

This document has been made available through Purdue e-Pubs, a service of the Purdue University Libraries. Please contact epubs@purdue.edu for additional information. 


\section{Direct Simulation of Transport in Open-Cell Metal Foam}

\section{Shankar Krishnan} Jayathi Y. Murthy

\section{Suresh V. Garimella}

\author{
NSF Cooling Technologies Research Center, \\ School of Mechanical Engineering, \\ Purdue University, \\ West Lafayette, IN 47907
}

Flows in porous media may be modeled using two major classes of approaches: (a) a macroscopic approach, where volume-averaged semiempirical equations are used to describe flow characteristics, and (b) a microscopic approach, where small-scale flow details are simulated by considering the specific geometry of the porous medium. In the first approach, small-scale details are ignored and the information so lost is represented in the governing equations using an engineering model. In the second, the intricate geometry of the porous structures is accounted for and the transport through these structures computed. The latter approach is computationally expensive if the entire physical domain were to be simulated. Computational time can be reduced by exploiting periodicity when it exists. In the present work we carry out a direct simulation of the transport in an open-cell metal foam using a periodic unit cell. The foam geometry is created by assuming the pore to be spherical. The spheres are located at the vertices and at the center of the unit cell. The periodic foam geometry is obtained by subtracting the unit cell cube from the spheres. Fluid and heat flow are computed in the periodic unit cell. Our objective in the present study is to obtain the effective thermal conductivity, pressure drop, and local heat transfer coefficient from a consistent direct simulation of the open-cell foam structure. The computed values compare well with the existing experimental measurements and semiempirical models for porosities greater than $94 \%$. The results and the merits of the present approach are discussed. [DOI: 10.1115/1.2227038]

\section{Introduction}

Metal foams are a class of materials with low densities and novel physical, mechanical, thermal, electrical, and acoustic properties. They offer great potential for use as lightweight structures, and for energy absorption and thermal management applications [1]. Metal foams can offer effective solutions to many thermal management problems because of their large surface area to volume ratio and high permeability to fluids [2-4].

Flows in porous media may be modeled using two major approaches: (a) a macroscopic approach, where volume-averaged semiempirical equations are used to describe flow characteristics, and $(b)$ a microscopic approach, where the small-scale flow details are simulated by considering the specific geometry of the porous medium. In the former approach, small-scale details are ignored and the information so lost is represented in the governing equations using an engineering model. In the latter approach, the intricate geometry of the porous structures is accounted for and the flow through these structures is computed. The latter approach is computationally expensive if the entire physical domain were to be simulated. Computational time can be reduced by exploiting periodicity in situations where periodicity is obtained.

Resolution of flow and heat transfer at the pore scale is necessary for a number of reasons when modeling metal foams. Detailed modeling of pore-scale heat transfer has been used to yield the effective thermal conductivity of the foam for situations with no flow. Existing models have frequently used idealized (and approximate) geometries assuming one-dimensional conduction heat transfer with a free parameter that is adjusted to match experimental results [5,6]. Another use for pore-scale models is to better characterize the pressure drop and local heat transfer coefficient during flow and convective heat transfer through the foam. Though there have been a few studies that take this approach $[7,8]$, the range of Reynolds numbers considered does not ad-

Contributed by the Heat Transfer Division of ASME for publication in the JouRNal of Heat Transfer. Manuscript received July 6, 2005; final manuscript received January 9, 2006. Review conducted by N. K. Anand. Paper presented at the 2005 ASME International Mechanical Engineering Congress (IMECE2005), November 5-11, 2005, Orlando, Florida, USA. equately cover metal-PCM systems where the pore Reynolds numbers are very small $(\operatorname{Re}<1)[9]$. The geometric representation of the foam varies greatly in the literature. Past investigators have represented the open-celled foam structure using (i) simple cubic unit cells consisting of slender circular cylinders [3], (ii) cubic unit cells consisting of square cylinders [10], (iii) truncated tetrakaidecahedron unit cells with triangular strands (fibers) [7], and (iv) a Weaire-Phelan unit cell [8]. Results from these models have included effective thermal conductivity and pressure drop calculations, but no information has been reported on local heat transfer. The local heat transfer coefficients are very important for closing (coupling) the solid and fluid energy equations in the twomedium volume-averaged models [9,11-13]. Also, the unit cell used to predict effective thermal conductivity is frequently different from the ones used for flow calculations [14]. Our objective in the present study is to obtain the effective thermal conductivity, pressure drop, and local heat transfer coefficient from a consistent direct simulation of the open-cell foam structure.

\section{Foam Geometry}

A three-dimensional periodic module is identified for the direct simulation of open-cell foams. The geometry chosen should be space filling and should have minimum surface energy. This is required because of the nature of the foaming process. A popular method for foaming metals such as aluminum is by blowing a foaming gas through molten metal with ceramic particles (used for stabilization) from below [1]. The gas bubbles developed are free to move around and pack themselves. The liquid metal accumulates at the interstices of the bubbles. For the process to reach a steady state, the bubbles must attain an equilibrium, i.e., a minimum surface energy state. Once the molten foam is solidified, the open-cell foam is rolled into sheets or into any other desired form [1]. Until recently, the body-centered-cubic (BCC) structure (similar to Kelvin's tetrakaidecahedron unit cell $[15,16])$, has been shown to have minimum surface-area to volume ratio compared to all other space filling structures [17]. Figure 1 shows a schematic diagram of a BCC unit cell. Though other choices such as the Weaire-Phelan (WP) unit cell are possible to model, a BCC unit cell model is used here for simplicity. 


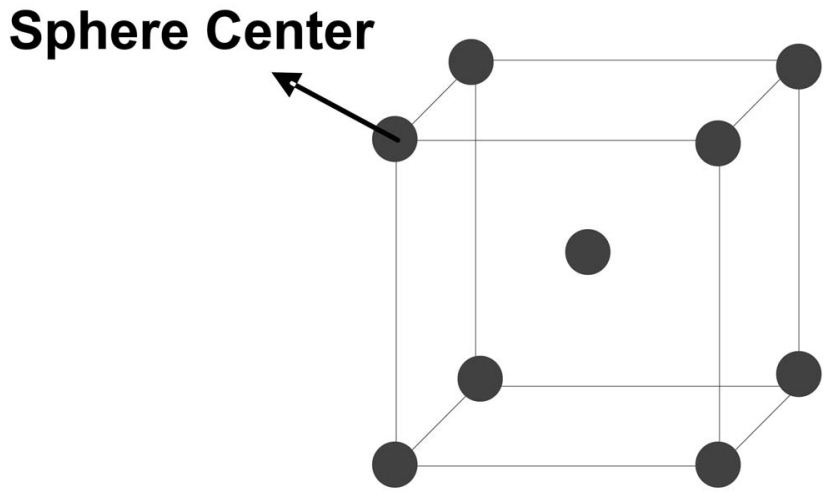

Fig. 1 Schematic of the body-centered-cubic model

In the present approach for foam geometry creation, the shape of the pore is assumed to be spherical and spheres of equal volumes are located at the vertices of the cell and at the center of the unit cell. The ideal periodic foam geometry is obtained by subtracting the unit cell cube from the spheres located at the body centers and vertices of the cube, as shown in Fig. 2. For open-cell structures, the sphere radius must be larger than half the side of the cube. The cross section of the foam ligaments are convex triangles (Plateau borders) and they all meet at symmetric tetrahedral vertices [17]. It may be noted that there is a nonuniform distribution of metal mass along the length of the ligament with more mass accumulating at the vertices (nodes), resulting in a thinning at the center of the ligament, as experimentally observed in foam samples by many authors $[1,6,14,18]$. Figure 3 shows some sample open-cell structures. The distinguishing feature of this approach is that (i) the geometry creation is simple; (ii) it captures many of the important features of real foams; and (iii) meshing of the geometry is easier compared to the approach carried out in [8] for modeling pressure drop. In [8], the foam was represented by an ideal Weaire-Phelan unit-cell obtained using Surface Evolver, a surface minimization software package. The idealized geometry was exported to a mesh generation program. After a series of post-processing steps on the geometry obtained from Surface Evolver, an unstructured volume mesh was generated for CFD calculations.

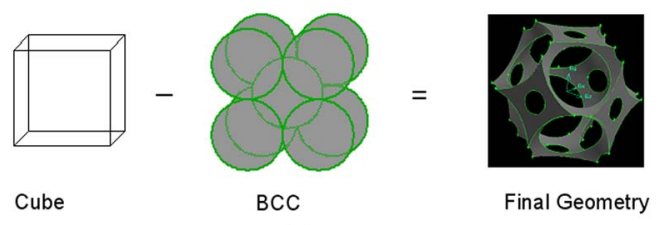

(a)

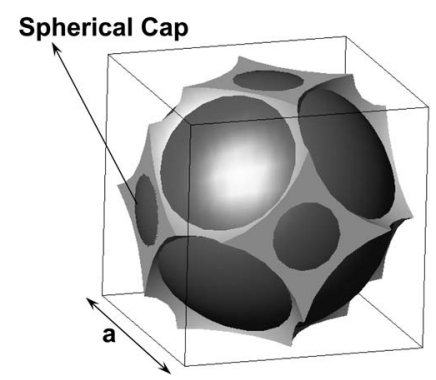

(b)

Fig. 2 Schematic diagram of (a) the geometry creation and (b) a periodic unit cell
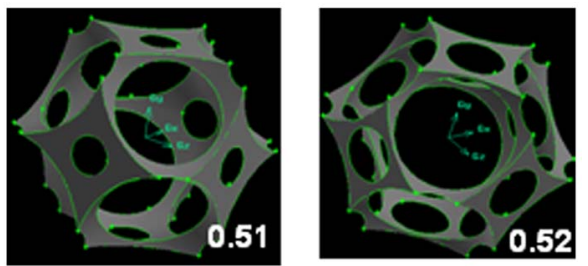

(a)

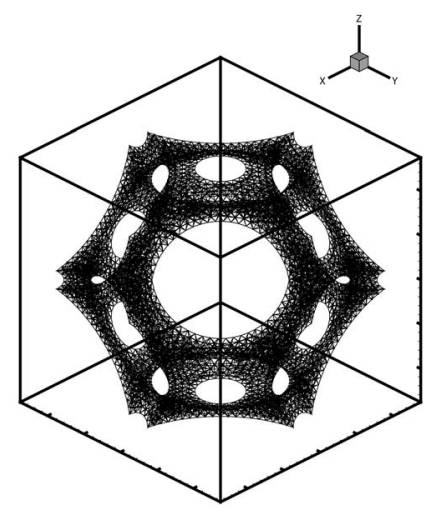

(b)

Fig. 3 Sample images of (a) the Representative Elementary Volume (REV) and (b) the computational mesh of the solidfoam (fluid zone grid points are omitted for clarity)

The expression for the porosity of the periodic module and the fluid inlet area of the periodic face can be obtained by accounting for the overlapping sphere volumes and circle areas, respectively. The intersection volume (lens volume) between two overlapping spheres is given by the relation

$$
V_{\mathrm{int}}=\frac{\pi}{12}(4 R+s)(2 R-s)^{2},
$$

where $\mathrm{s}$ is the center-to-center distance between the in-line spheres and $R$ is the radius of the sphere. The body-centered sphere intersects with eight spheres on the vertices of the cube and hence the volume of the sphere at the body center of the cube is

$$
V_{b c}^{\prime}=\frac{4 \pi}{3} R^{3}-8\left(\frac{V_{\mathrm{int}}}{2}\right)
$$

In addition to the sphere at the body center, there is one additional sphere volume contributed by the eight segments of the sphere at the vertices. Hence the total sphere volume not accounting for the spherical caps (see Fig. 2(b)) at the intersection between the face of the cube (plane) and the spheres is twice the $V_{\mathrm{bc}}^{\prime}$ given in Eq. (2). The volume occupied by the spherical cap (the protruding volume from the unit cube for sphere diameter larger than length of the cube) due to a sphere intersecting a plane is given by the expression

$$
V_{\mathrm{sc}}^{\prime}=\frac{\pi}{3}(R-a / 2)^{2}(2 R+a / 2)
$$

There are six spherical caps for the six corresponding faces of the cube and hence the volume of the fluid space in the cube is given by the expression

$$
V_{f}^{\prime}=2\left(\frac{4 \pi}{3} R^{3}-\frac{\pi}{3}(4 R+s)(2 R-s)^{2}-2 \pi(R-a / 2)^{2}(2 R+a / 2)\right)
$$

and, hence, the porosity is given by the relation 


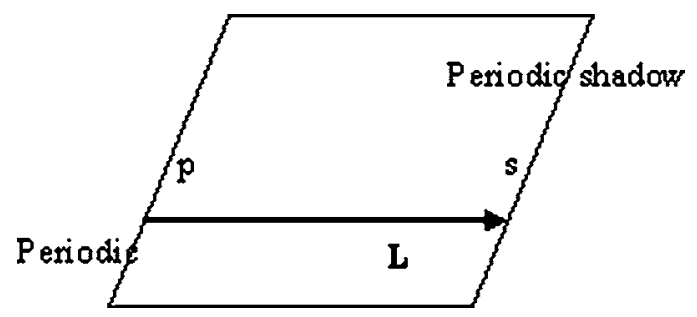

Fig. 4 Schematic illustration of a periodic domain

$$
\begin{aligned}
\varepsilon & =\frac{V_{f}^{\prime}}{V} \\
& =\frac{2\left(\frac{4 \pi}{3} R^{3}-\frac{\pi}{3}(4 R+s)(2 R-s)^{2}-2 \pi(R-a / 2)^{2}(2 R+a / 2)\right)}{a^{3}}
\end{aligned}
$$

In the above expressions, $a$ is the length of the side of the unit cell. Similarly, the inlet face area for the fluid can be obtained by subtracting the circle area and the intersection area between the sphere and the plane from the face area. The inlet face area is given by the expression

$$
A_{\mathrm{in}, f}=\pi R^{2}-2\left[2 R^{2} \cos ^{-1}\left(\frac{s}{2 R}\right)-\frac{1}{2} s \sqrt{4 R^{2}-s^{2}}\right]+\pi\left(\frac{4 R^{2}-a^{2}}{4}\right)
$$

It should be noted that the center-to-center distance $(\sqrt{3} \mathrm{a} / 2)$ for the volume (porosity) calculation is different from the center-tocenter distance $(a / 2)$ for the area calculation. An effective pore diameter was extracted, setting Eq. (4) equal to the area of an equivalent circle. It should be noted that exactly at a sphere radius of 0.5 , the BCC structure ceases to be a complete open structure, and the corresponding porosity at this condition is 0.94 . The implications of this porosity on model fidelity will be discussed later in this paper.

\section{Mathematical and Numerical Modeling}

Flow and Temperature Periodicity. Consider a module with periodic boundaries separated by a constant translation vector $(\vec{L})$, as shown in Fig. 4. For simplicity, a two-dimensional domain is shown. This module represents one of a series of periodic modules translated by $\vec{L}$. It should be noted that there may be other periodic boundaries in the module, but there is no net inflow through any of these boundaries. For periodic boundaries, according to [19], the following relationship holds for the velocity and the pressure at any position $\vec{r}$ :

$$
\begin{gathered}
u_{i}(\vec{r})=u_{i}(\vec{r}+\vec{L})=u_{i}(\vec{r}+2 \vec{L})=\cdots \\
P(\vec{r})-P(\vec{r}+\vec{L})=P(\vec{r}+\vec{L})-P(\vec{r}+2 \vec{L})=\cdots
\end{gathered}
$$

For periodic flows, the pressure gradient can be divided into two components - the gradient of the periodic component, $\partial \hat{p} / \partial x_{i}$, and the gradient of a linearly varying component, $\left(\partial \bar{p} / \partial x_{i}\right) \vec{e}_{L}$ :

$$
\frac{\partial P}{\partial x_{i}}=-\frac{\partial \bar{p}}{\partial x_{i}} \vec{e}_{L, i}+\frac{\partial \hat{p}}{\partial x_{i}}
$$

where $e_{L, i}$ is the $i$ th component of the unit vector in the direction $\vec{L}$.

For given heat-flux boundary conditions, the shape of the temperature field becomes constant from module to module. Consequently, the periodic condition for the temperature is given by

$$
T(\vec{r})-T_{b}(\vec{r})=T(\vec{r}+\vec{L})-T_{b}(\vec{r}+\vec{L})=T(\vec{r}+2 \vec{L})-T_{b}(\vec{r}+2 \vec{L})=\cdots
$$

Here the bulk temperature $T_{b}$ is defined as

$$
\frac{\iint_{A}\left|u_{i} e_{L, i}\right| T \mathrm{~d} A}{\iint_{A}\left|u_{i} e_{L, i}\right| d A}=0
$$

where $A$ is the area of the cross section.

Governing Equations. The governing flow and heat transfer equations for periodic fully developed incompressible, steady flow of a Newtonian fluid are $[19,20]$

$$
\begin{gathered}
\frac{\partial}{\partial x_{i}}\left(\rho u_{i}\right)=0 \\
\frac{\partial}{\partial x_{j}}\left(\rho u_{i} u_{j}\right)=\frac{\partial p}{\partial x_{i}}+\frac{\partial}{\partial x_{j}}\left(\mu \frac{\partial u_{i}}{\partial x_{j}}\right)_{i}-\frac{\partial \bar{p}}{\partial x_{i}} e_{L, i} \\
\frac{\partial}{\partial x_{i}}\left(\rho u_{i} C_{p} T\right)=\frac{\partial}{\partial x_{i}}\left(k \frac{\partial T}{\partial x_{i}}\right)
\end{gathered}
$$

The above equations assume that the flow is thermally and hydrodynamically fully developed. In Eq. (6), the terms involving $\partial^{2} / \partial x^{2}$ have been included to account for the large local streamwise gradients that may occur in periodically fully developed flows. The quantity $\partial \bar{p} / \partial x_{i}$ in Eq. (6) is assigned a priori, and controls the mass flow rate through the module, and hence the pore Reynolds number. In order to sustain periodicity, all fluid properties are assumed to be independent of temperature. It should be noted that on the solid bounding walls a no-slip boundary condition is imposed for the velocities and a constant heat flux is specified for the energy equation. Details of the mathematical model are available in [19]. An extensive treatment of the numerical method for the periodic flow and heat transfer on unstructured meshes along with the implementation is given in [20].

The periodic unit cell geometry was created using the commercial software GAMBIT [21]. The geometry was meshed using hybrid (tetrahedral and hexagonal) elements in GAMBIT by specifying the minimum edge length. The mesh so created was exported to the commercial code FLUENT [22] for flow simulations. A second-order upwind scheme was used for the flow and heat transfer calculations. A colocated pressure-velocity formulation in conjunction with the SIMPLE algorithm was used for obtaining the velocity fields, and the linearized systems of equations are solved using an algebraic multigrid algorithm. Details of the numerical method may be found in [23]. The calculations were terminated when the (scaled) residual [22] had dropped below $10^{-7}$ for all governing equations.

Grid independence of the solution for the meshes used in the present simulations was established. A pore Reynolds number of 20 , a Prandtl number of 0.71 , and a porosity of 0.965 were used for this set of calculations. Grid sensitivity was tested on three different grids: grid 1 (106,520 cells), grid 2 (188,885 cells), and grid 3 (383,230 cells). For grid 1, deviations of $2.6 \%$ and $0.5 \%$ in the friction factor and Nusselt number were found with respect to grid 3. For grid 2, the corresponding deviations with respect to grid 3 were $0.9 \%$ and $0.4 \%$. The calculations reported in this paper were therefore performed on grid 2.

\section{Results and Discussion}

Effective Thermal Conductivity. In addition to the friction factor and Nusselt number results described above, the effective thermal conductivity of the foam is computed by considering heat conduction through the solid structure, in the absence of fluid flow. The effective thermal conductivity is computed numerically 
by solving the conduction heat transfer equation $(\nabla \cdot k \nabla T=0)$. Only a single periodic module is used in the analysis. Using the periodicity assumption, each module in the heat flow direction experiences an equal temperature drop, i.e.,

$$
\nabla T(\vec{r})=\nabla T(\vec{r}+\vec{L})=\nabla T(\vec{r}-\vec{L})
$$

The numerical implementation of this type of periodic condition in the unstructured finite volume framework is described in [24]. Computations are performed by choosing an arbitrary temperature drop $\Delta T$ across the module in the heat flow direction and finding the resulting heat transfer rate at the periodic boundaries from the simulation results. The effective thermal conductivity of the module is then given by the expression

$$
k_{e f f}=\frac{-\int_{A_{p}} \mathbf{J} \cdot \mathrm{d} \mathbf{A}}{\Delta T A_{p}}
$$

In the above equation, $\mathbf{J}$ is the diffusion flux vector at the periodic face obtained from the simulation, $d A$ is the outward pointing elemental face, area vector on the periodic face, and $A_{p}$ is the area of the periodic face. Computations are performed using a modified version of the commercial code FLUENT [22]. It is noted that conduction through the solid foam and the fluid are considered for the calculation of the effective thermal conductivity of the module.

Lemlich Theory. Lemlich [25] developed a theory to predict the electrical conductivity of a polyhedral liquid foam of high porosity. The electrical conduction is viewed as occurring only through the Plateau border (ligament in the case of solid foams) along its axis, and not through its periphery. He found that the effective electrical conductivity of the foam was related to the electrical conductivity of the liquid through the following relation:

$$
\sigma_{\text {eff }}=\sigma_{l} \frac{(1-\varepsilon)}{3}
$$

This expression can be used for the effective thermal conductivity of the solid foam by exploiting the analogy between Ohm's law and Fourier's law, so that

$$
k_{\mathrm{eff}}=k_{s} \frac{(1-\varepsilon)}{3}
$$

Figures 5(a) and 5(b) show the predicted effective thermal conductivity from the simulations as a function of porosity for an open cell foam saturated with air and water, respectively. Also plotted in Fig. 5 are measured experimental values $[6,26]$ and results from semiempirical models in the literature [6]. It can be seen from Fig. 6 that the present model compares well with the experiments (both air and water) and the other models for porosities above 0.94 . The foam geometry ceases to be "open" celled for porosities below 0.94 , as previously discussed. It may be recalled that the models of Boomsma and Poulikakos [5], Calmidi and Mahajan [6], and Bhattacharya et al. [14] employ an adjustable free parameter to match the experiments of Calmidi and Mahajan [6]. The computations in this paper employ no such adjustable parameter; here, the attempt is to directly compute the effective conductivity from a detailed description of the foam geometry. It may be noted, however, that deviations from experimental data reflect the inadequacy of the present geometric model at lower porosities.

The Lemlich theory predicts the thermal conductivity values well when the interstitial fluid is air (Fig. 5(a)), but is less successful with water saturation (Fig. 5(b)). This deviation for water is primarily due to the assumption of negligible heat exchange to the interstitial fluid in the model, and also due to the ignored contribution of nodal resistance at the tetrahedral vertices. In the case of water (whose thermal conductivity is an order of magnitude higher than that of air) the heat exchange between the foam
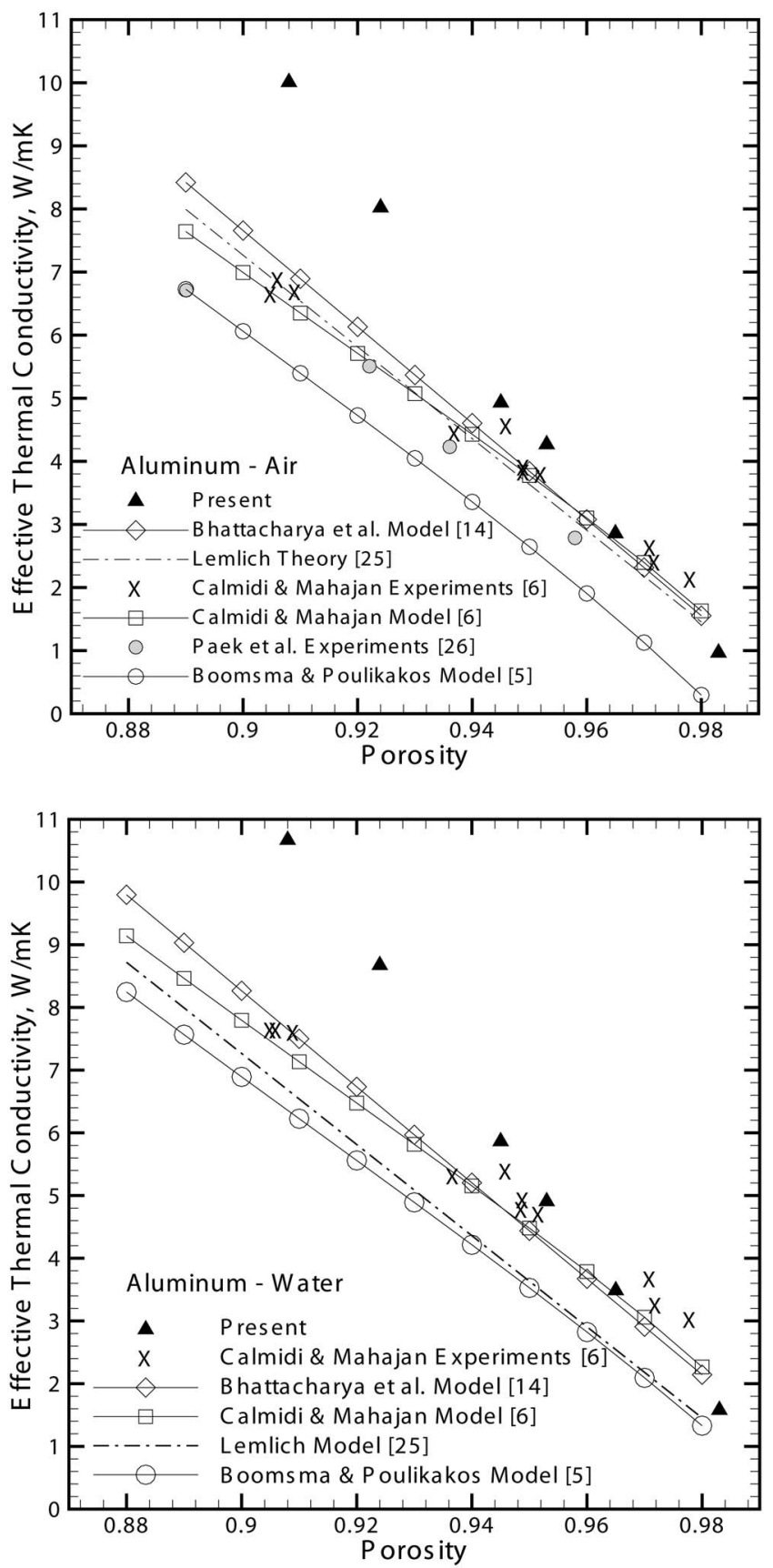

Fig. 5 Effective thermal conductivity as a function of porosity for aluminum foam saturated with (a) air and (b) water. The thermal conductivity values used for aluminum, air, and water are $218,0.0265$, and $0.613 \mathrm{~W} / \mathrm{mK}$, respectively [5]

ligament and interstitial fluid is significant. Equation (10) may thus be used for very satisfactory order-of-magnitude estimates of effective thermal conductivity of open cell foams.

Pressure-Drop and Heat Transfer Coefficient. For the calculations presented in this section, a constant heat flux boundary condition was imposed on the ligament walls. Hence, conduction through the ligaments of the foam is neglected. The streamwise diffusion term is retained in the momentum and energy equations which govern the generalized fully developed regime [19].

For a fluid flowing through a porous medium, boundary layer growth is significant only over an axial length of order $\left(\rho \mathrm{Ku}_{\mathrm{D}}\right) / \mu$, where $u_{\mathrm{D}}$ is $(\mathrm{K} / \mu) \mathrm{dP} / \mathrm{dx}$. Similarly, the thermal boundary layer 


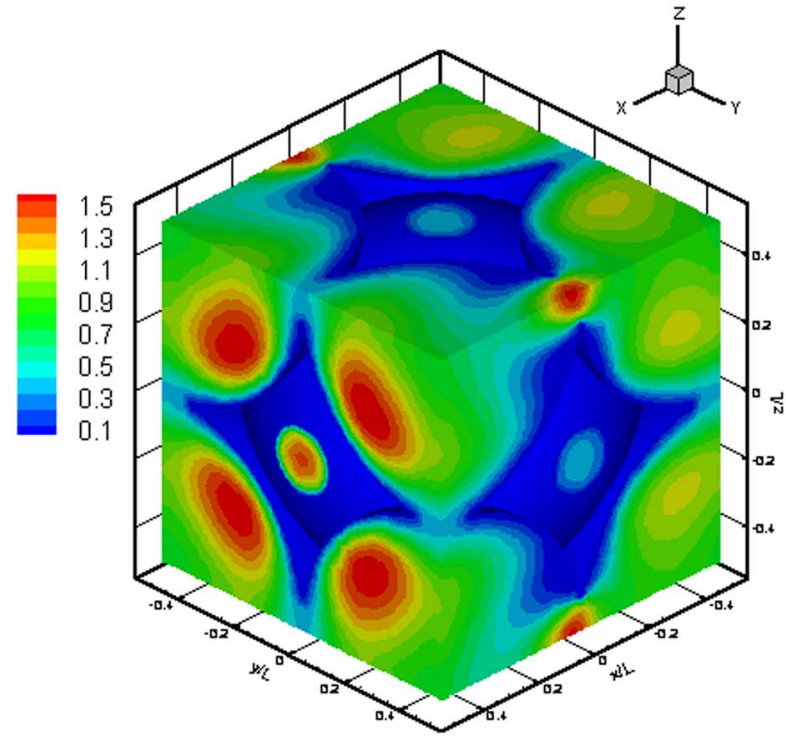

(a)

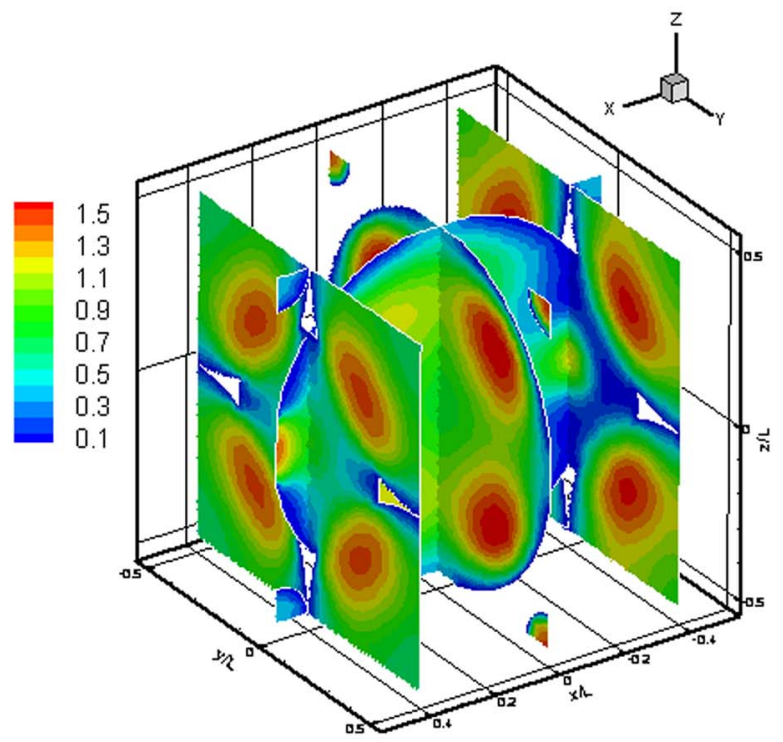

(b)

Fig. 6 Predicted results for (a) dimensionless velocity field, and $(b)$ dimensionless velocity field at different locations $(x / L$ $=-0.4,0.0,0.4$, and $y / L=0$ )

development length is on the order of $\mathrm{Ku}_{\mathrm{D}} / \alpha$. The flow penetration is usually on the order of $\sqrt{ } \mathrm{K}$, the characteristic length scale. It should be noted that unlike packed beds of spheres, the porosity and permeability for open-cell foams are constant even close to the solid boundary, i.e., the porosity does not change near the boundaries. In this section, a representative case of porosity $=0.965$ with air being the interstitial fluid is explained first. In the later part of the section we discusses the predicted variation of the friction factor and local Nusselt number.

Figure 6 shows the predicted dimensionless $u$-velocity field normalized using the mean velocity for a porosity of 0.965 . The Reynolds number based on the effective diameter of the pore is 50 and the Prandtl number is 0.7. The effective diameter for all the computations is obtained by setting Eq. (4) equal to that of the area of a circle and backing out the effective diameter. Figure $6(b)$ shows the dimensionless velocity field on slices at discrete locations along the axial direction of flow. Also plotted in Fig. $6(b)$ is

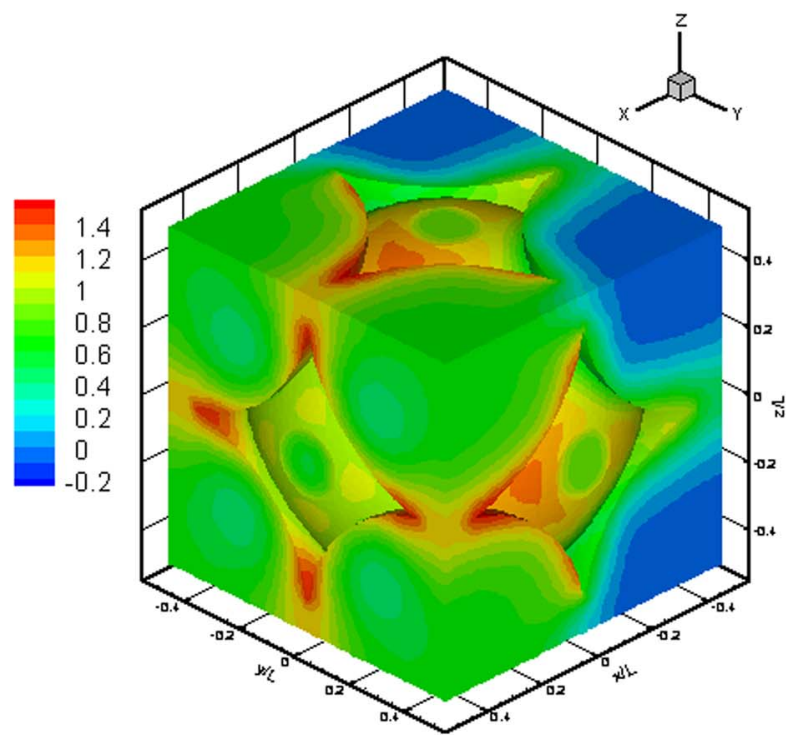

(a)

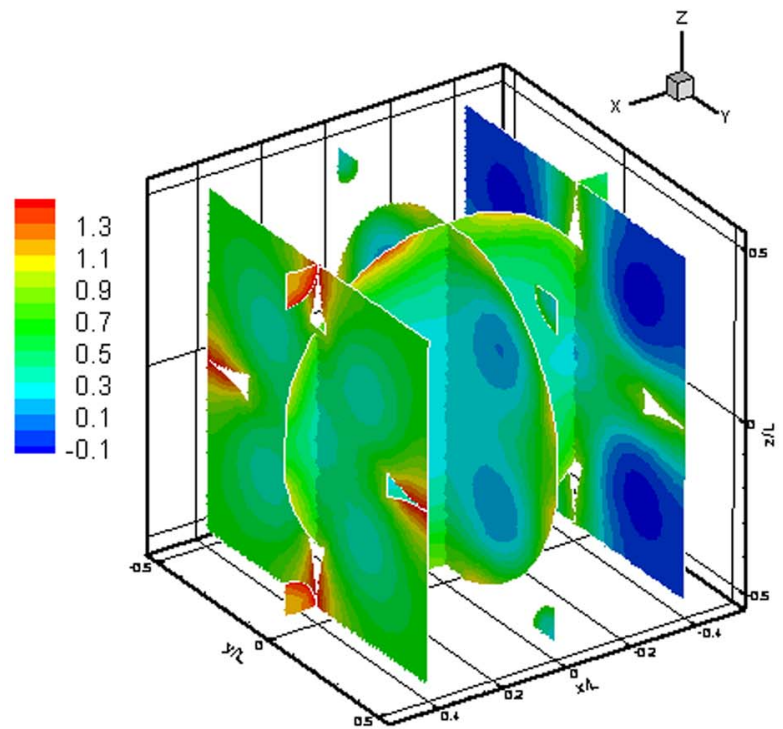

(b)

Fig. 7 Predicted results for (a) dimensionless temperature field and $(b)$ dimensionless temperature maps at different locations $(x / L=-0.4,0$ and 0.4 , and $y / L=0)$

the velocity field at $y / L=0$ to illustrate the axial flow. The flow enters at the periodic inlet, $x / L=-0.5$. Periodic conditions are specified on all the boundary faces of the cubic unit cell. The solid boundaries are demarcated in white in the figure. From Fig. 6 the boundary layer development at the solid boundaries can be seen. Due to the resistance offered by the foam ligaments, the mean velocities are higher in some regions. Figure 7 shows the dimensionless temperature distribution $\left[\left(T-T_{b}\right) /\left(\bar{T}_{s}-T_{b}\right)\right]$ in the fluid for a porosity value of 0.965 . As expected, the dimensionless temperature at the periodic inlet is low and thermal boundary layers can be clearly seen in Fig. $7(b)$. For Pe $>1$, thermal dispersion effects become important $[27,28]$. The calculation of thermal dispersion conductivity is beyond the scope of this paper and is being considered in ongoing work.

Figure 8 shows the predicted normalized permeability of the foam as a function of porosity. Also shown are the reported ex- 


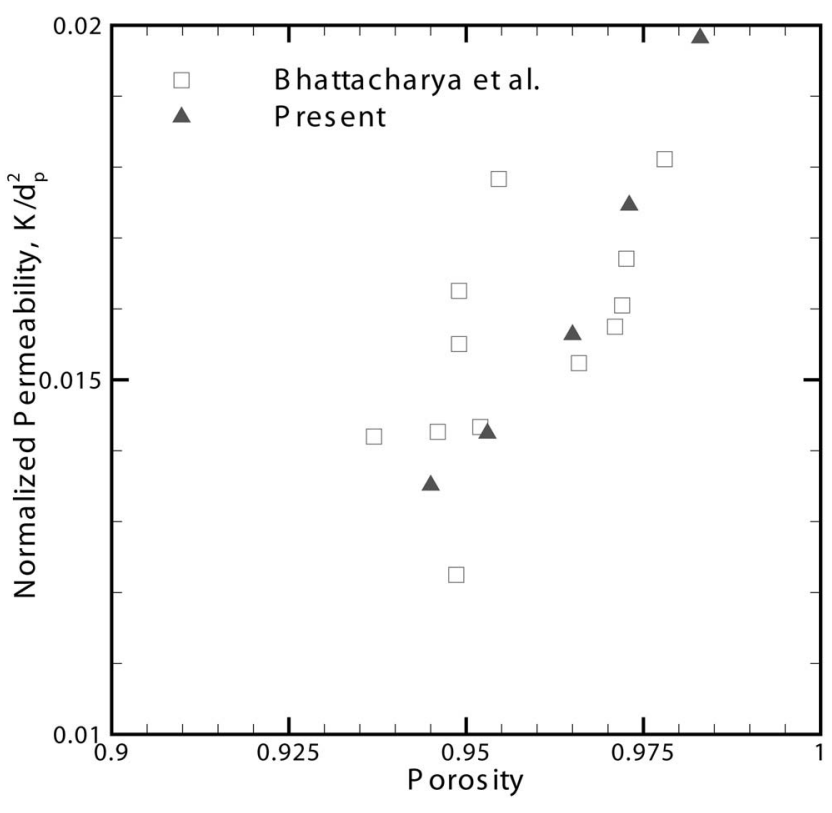

Fig. 8 Predicted normalized permeability of the foam as a function of the porosity of the foam. Also plotted are experimental data points from Bhattacharya et al. [14].

perimental measurements from Bhattacharya et al. [14]. The permeability was normalized with the mean pore diameter of the open cell. The permeability was calculated from Darcy's law, $K$ $=-\left(\mu \mathrm{u}_{\text {mean }}\right) /\left(\partial \bar{p} / \partial x_{i}\right)$ where the $u_{\text {mean }}$ was obtained from the specified periodic inlet mass flow rate and using Eq. (4). The pressure drop was obtained as an output from the simulations. It should be noted that the reported permeability values are averaged values over a Reynolds number range of 1 to 10, i.e., in the linear Darcy regime.

Friction factors for the different cases considered are shown in Fig. 9 for porosities greater than 0.94. Also plotted in Fig. 9 are the experimental correlations from Paek et al. [26] and Vafai and Tien [29]. The friction factor is defined as

$$
f=\frac{\left(-\frac{\partial \bar{p}}{\partial x_{i}}\right) \sqrt{K}}{\rho u_{\text {mean }}^{2}}
$$

and in the Darcy regime, the friction factor $(f)$ scales as the inverse of the modified Reynolds number based on the flow penetration length $\left(f=1 / \mathrm{Re}_{K}\right)$. From Fig. 9, it can be seen that for the porosity and modified Reynolds number $\left(\operatorname{Re}_{K} \approx 1-10\right)$ ranges considered in this study, the flow of both air and water through the foam is still in the Darcy regime. Deviations from $1 / \mathrm{Re}_{K}$ behavior were observed near a modified Reynolds number of approximately 20 for both air and water.

The Nusselt number for the foam was also calculated for the different cases considered and is defined as

$$
\mathrm{Nu}=\frac{h D}{k_{f}}=\frac{q^{\prime \prime} D}{k_{f}\left(\bar{T}_{s}-T_{b}\right)}
$$

In the above equation $\bar{T}_{s}$ is the averaged temperature of the foam. Figure 10 shows the predicted Nusselt number as a function of the square root of the Peclet number. This scale is readily obtained by balancing the convective and axial diffusive fluxes. Also plotted in Fig. 10 is the correlation from Calmidi and Mahajan computed for air and for a porosity of 0.973 [30]. While their original correlation was based on the fiber diameter, it is rescaled here in terms of the mean pore diameter, with 0.00402 and $0.0005 \mathrm{~m}$ as the fiber and pore diameters, respectively [30]. The curves for air and wa-

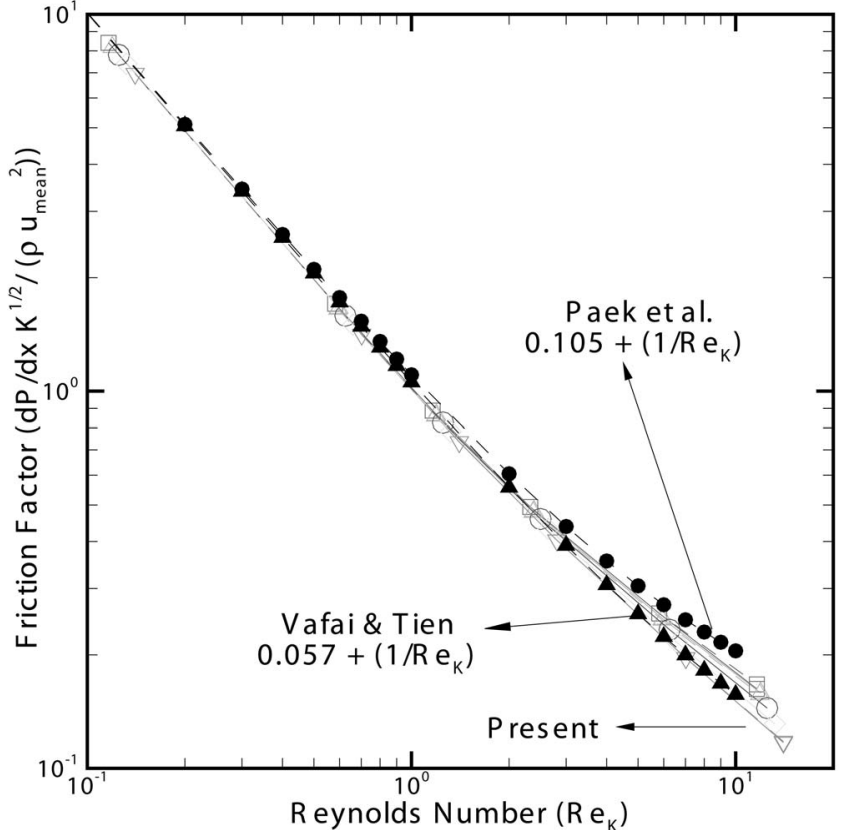

Fig. 9 Predicted friction factor as a function of Reynolds number based on the flow penetration length $\left({ }_{V} K\right)$. Also plotted are experimental correlations from Paek et al. [26] and Vafai and Tien [29]. Symbols are defined in Fig. 10.

ter, respectively, are seen to collapse to a single line (unique slope) for $\mathrm{Pe}_{d}<30$, but they have different slopes due to the difference in the Prandtl number.

\section{Conclusions}

In this paper we present a consistent approach to the simulation of open-cell foam geometries for the study of effective thermal conductivity, friction factor, and Nusselt number. A BCC unit cell

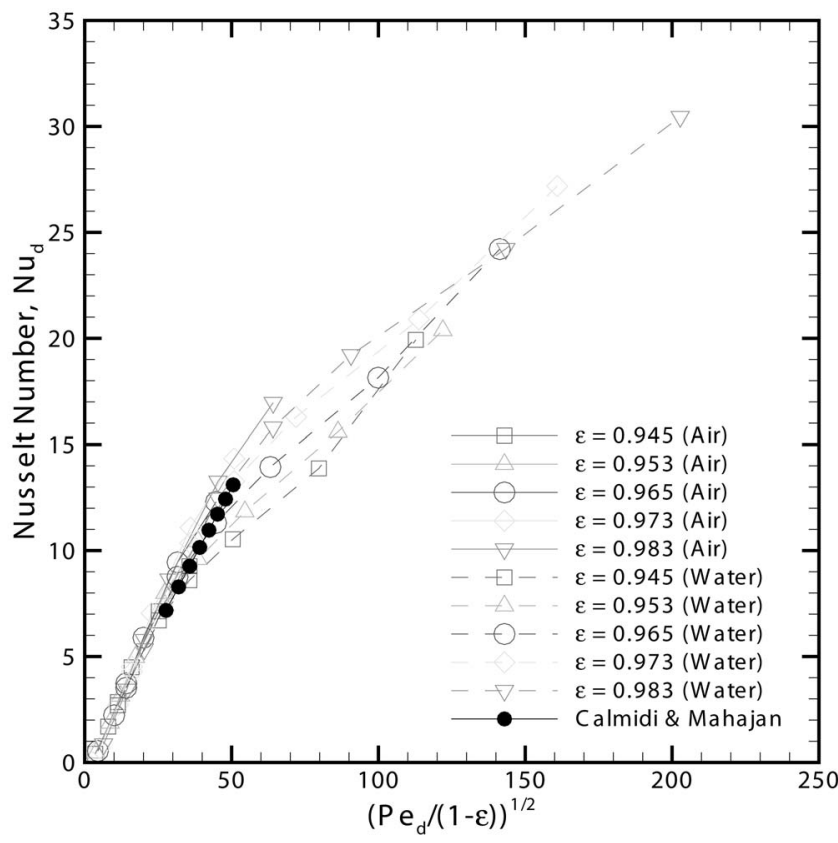

Fig. 10 Predicted Nusselt number based on effective diameter of the pore as a function of the square root of the Peclet number $\left[\mathrm{Re}_{d} \operatorname{Pr} /(1-\varepsilon)\right]$. Also plotted is the correlation from Calmidi and Mahajan [30]. 
model is used with no adjustable geometric parameters to match experiments for all three computations, unlike previous published work. For porosities higher than $94 \%(\varepsilon>0.94)$, the predicted effective thermal conductivity, friction factor, and Nusselt number from the simulations compare reasonably well with the existing experimental and semiempirical models. However, the predicted effective thermal conductivity departs from measurements for low-porosity foams that are no longer open-celled; alternative geometric descriptions must be investigated at these lower porosities. For $\varepsilon>0.94$, the effective thermal conductivity scales as $k_{s}(1-\varepsilon) / 3$ for the range of porosities investigated, while the friction factor and Nusselt number scale as $1 / \mathrm{Re}_{K}$ and $[\mathrm{Pe} /(1$ $-\varepsilon)]^{1 / 2}$, respectively. It is expected that thermal dispersion effects will be significant for $\mathrm{Pe}>1[27,28]$. The present study may be extended to evaluate thermal dispersion effects, as is being done in ongoing work.

\section{Acknowledgment}

Support for this work from industry members of the Cooling Technologies Research Center, an NSF Industry/University Cooperative Research Center (www.ecn.purdue.edu/CTRC), is gratefully acknowledged. The authors wish to thank Dr. Dawei Sun for his help with AutoCAD.

\section{Nomenclature}

$$
\begin{aligned}
a & =\text { edge length of the unit cell, } \mathrm{m} \\
A & =\text { area, } \mathrm{m}^{2} \\
C_{p} & =\text { specific heat capacity, } J_{\mathrm{kg}^{-1} \mathrm{~K}^{-1}} \\
D & =\text { diameter of the pore, } \mathrm{m} \\
\mathrm{Da} & =\text { Darcy number } \\
f & =\text { friction factor } \\
h & =\text { heat transfer coefficient, } \mathrm{Wm}^{-2} \mathrm{~K}^{-1} \\
J & =\text { diffusion flux vector, } \mathrm{m}^{2} \mathrm{~s}^{-1} \\
K & =\text { permeability, } \mathrm{m}^{2} \\
k & =\text { thermal conductivity, } \mathrm{Wm}^{-1} \mathrm{~K}^{-1} \\
L & =\text { length of the periodic module, } \mathrm{m} \\
\mathrm{Nu} & =\text { Nusselt number } \\
q \prime \prime & =\text { heat flux, Wm } \\
\mathrm{Pr} & =\text { Prandtl number } \\
\mathrm{Pe} & =\text { Peclet number } \\
R & =\text { radius of the pore, } \mathrm{m} \\
\mathrm{Re} & =\text { Reynolds number } \\
s & =\text { center-to-center distance, } \mathrm{m} \\
T & =\text { temperature, } \mathrm{K} \\
t & =\text { time, } \mathrm{s} \\
u, v, w & =\text { velocities along } x, y, z \text { directions, } \mathrm{ms}^{-1} \\
V & =\text { volume, m } \\
x, y, z & =\text { Cartesian coordinates }
\end{aligned}
$$

\section{Greek Symbols}

$$
\begin{aligned}
\alpha & =\text { thermal diffusivity, } \mathrm{m}^{2} \mathrm{~s}^{-1} \\
\varepsilon & =\text { porosity } \\
\mu & =\text { dynamic viscosity, } \mathrm{kg} \mathrm{m}^{-1} \mathrm{~s}^{-1} \\
\rho & =\text { density, } \mathrm{kg} \mathrm{m}^{-3}
\end{aligned}
$$

\section{Superscripts}

$$
- \text { = average or mean }
$$

\section{Subscripts}

$$
\begin{aligned}
b & =\text { bulk } \\
\mathrm{bc} & =\text { body center } \\
\mathrm{D} & =\text { Darcian } \\
\text { eff } & =\text { effective } \\
f & =\text { fluid }
\end{aligned}
$$

$$
\begin{aligned}
\text { in } & =\text { inlet } \\
\text { int } & =\text { intersection } \\
s & =\text { solid } \\
\mathrm{sa} & =\text { surface area } \\
\mathrm{sc} & =\text { spherical cap }
\end{aligned}
$$

\section{References}

[1] Ashby, M. F., Evans, A., Fleck, N. A., Gibson, L. J., Hutchinson, J. W., and Wadley, H. J. G., 2000, Metal Foams: A Design Guide, ButterworthHeinemann, Boston.

[2] Boomsma, K., Poulikakos, D., and Zwick, F., 2003, "Metal Foams as Compact High Performance Heat Exchangers," Mech. Mater., 35, pp. 1161-1176.

[3] Lu, T. J., Stone, H. A., and Ashby, M. F., 1998, "Heat Transfer in Open-Cell Metal Foams," Acta Mater., 46, pp. 3619-3635.

[4] Ozmat, B., Leyda, B., and Benson, B., 2004, "Thermal Applications of OpenCell Metal Foams," Mater. Manuf. Processes, 19, pp. 839-862.

[5] Boomsma, K., and Poulikakos, D., 2001, "On the Effective Thermal Conductivity of a Three-Dimensionally Structured Fluid-Saturated Metal Foam," Int. J. Heat Mass Transfer, 44, pp. 827-836.

[6] Calmidi, V. V., and Mahajan, R. L., 1999, "The Effective Thermal Conductivity of High Porosity Fibrous Metal Foams," ASME J. Heat Transfer, 121, pp. 466-471.

[7] Fourie, J. G., and Du Plessis, J. P., 2002, "Pressure Drop Modeling in Cellular Metallic Foams," Chem. Eng. Sci., 57, pp. 2781-2789.

[8] Boomsma, K., Poulikakos, D., and Ventikos, Y., 2003, "Simulations of Flow Through Open Cell Foams Using an Idealized Periodic Cell Structure,” Int. J. Heat Fluid Flow, 24, pp. 825-834.

[9] Krishnan, S., Murthy, J. Y., and Garimella, S. V., 2005, "A Two-Temperature Model For Solid-Liquid Phase Change in Metal Foams," ASME J. Heat Transfer, 127, pp. 995-1004.

[10] DuPlessis, P., Montillet, A., Comiti, J., and Legrand, J., 1994, "Pressure Drop Prediction For Flow Through High Porosity Metallic Foams," Chem. Eng. Sci., 49, pp. 3545-3553.

[11] Krishnan, S., Murthy, J. Y., and Garimella, S. V., 2004, "A Two-Temperature Model For the Analysis of Passive Thermal Control Systems," ASME J. Heat Transfer, 126, pp. 628-637.

[12] Kaviany, M., 1995, Principles of Heat Transfer in Porous Media, SpringerVerlag, New York.

[13] Amiri, A., and Vafai, K., 1994, "Analysis of Dispersion Effects and NonThermal Equilibrium, Non-Darcian, Variable Porosity Incompressible Flow Through Porous Media," Int. J. Heat Mass Transfer, 37, pp. 939-954.

[14] Bhattacharya, A., Calmidi, A. A., and Mahajan, R. L., 2002, "Thermophysical Properties of High Porosity Metal Foams," Int. J. Heat Mass Transfer, 45, pp. $1017-1031$

[15] Thomson, W., 1887, "On the Division of Space With Minimum Partitional Area," Philos. Mag., 5, pp. 645-654.

[16] Weaire, D., 2001, "A Philomorph Looks at Foam," Proc. Am. Philos. Soc., 145, pp. 564-574.

[17] Phelan, R., Weaire, D., and Brakke, K., 1995, "Computation of Equilibrium Foam Structures Using the Surface Evolver," Exp. Math., 4, pp. 181-192.

[18] Dharmasena, K. P., and Wadley, H. N. G., 2002, "Electrical Conductivity of Open-Cell Metal Foams," J. Mater. Res., 17, pp. 625-631.

[19] Patankar, S. V., Liu, C. H., and Sparrow, E. M., 1977, "Fully Developed Flow and Heat Transfer in Ducts Having Streamwise-Periodic Variations of CrossSectional Area," ASME J. Heat Transfer, 99, pp. 180-186.

[20] Murthy, J. Y., and Mathur, S., 1997, "Periodic Flow and Heat Transfer Using Unstructured Meshes," Int. J. Numer. Methods Fluids, 25, pp. 659-677.

[21] Fluent Inc., User's Guide for GAMBIT 2.0, 2002.

[22] Fluent Inc., User's Guide for FLUENT 6.0, 2002.

[23] Mathur, S. R., and Murthy, J. Y., 1997, "A Pressure-Based Method for Unstructured Meshes," Numer. Heat Transfer, Part B, 31, pp. 195-216.

[24] Kumar, S., and Murthy, J. Y., 2005, "A Numerical Technique For Computing Effective Thermal Conductivity of Fluid-Particle Mixtures," Numer. Heat Transfer, Part B, 47, pp. 555-572.

[25] Lemlich, R., 1978, "A Theory For the Limiting Conductivity of Polyhedral Foam at Low Density," J. Colloid Interface Sci., 64, pp. 107-110.

[26] Paek, J. W., Kang, B. H., Kim, S. Y., and Hyun, J. M., 2000, "Effective Thermal Conductivity and Permeability of Aluminum Foam Materials," Int. J. Thermophys., 21, pp. 453-464.

[27] Koch, D. L., and Brady, J. F., 1985, "Dispersion in Fixed Beds," J. Fluid Mech., 154, pp. 399-427.

[28] Koch, D. L., and Brady, J. F., 1985, "The Effective Diffusivity of Fibrous Media," AIChE J., 32, pp. 575-591.

[29] Vafai, K., and Tien, C. L., 1982, "Boundary and Inertia Effects on Convective Mass Transfer in Porous Media," Int. J. Heat Mass Transfer, 25, pp. 11831190.

[30] Calmidi, V. V., and Mahajan, R. L., 2000, "Forced Convection in High Porosity Metal Foams," ASME J. Heat Transfer, 122, pp. 557-565. 